\title{
Determination of solar cell parameters from its current-voltage and spectral characteristics
}

\author{
M. Tivanov ${ }^{\mathrm{a}}$, A. Patryn ${ }^{\mathrm{b}}$, N. Drozdov ${ }^{\mathrm{a}}$, A. Fedotov ${ }^{\mathrm{a}, *}$, \\ A. Mazanik ${ }^{\mathrm{a}}$ \\ ${ }^{a}$ Belarusian State University, F. Skaryna av. 4, 220050 Minsk, Belarus \\ ${ }^{\mathrm{b}}$ Technical University of Koszalin, Sniadeckich str. 2, 75-453 Koszalin, Poland
}

Received 15 May 2004; received in revised form 7 July 2004; accepted 10 July 2004

Available online 7 December 2004

\begin{abstract}
An algorithm for the calculation of solar cell parameters (series and parallel resistance, diode coefficient, reverse current density) calculation from its current-voltage characteristics at fixed illumination intensity is proposed. The possibility of determining the $\mathrm{p}-\mathrm{n}$ junction depth on the basis of spectral dependencies of diode photocurrent at different values of the applied bias voltage is shown.
\end{abstract}

(C) 2004 Elsevier B.V. All rights reserved.

Keywords: Solar cell parameters; p-n junction depth; $I-V$ characteristics; Spectral characteristics

\section{Introduction}

Significant elevation of solar cell (SC) efficiency can be achieved by optimization of physical and technological SC parameters and their exact correlation with sun spectrum. This is possible only with the creation of a nondestructive control system for SC parameters in all stages of SC manufacture. In our work we present different nondestructive procedures which allow us to determine series $R_{\mathrm{S}}$ and parallel $R_{\mathrm{P}}$ resistance of SC, diode coefficient $A$, reverse current $I_{0}$ and $\mathrm{p}-\mathrm{n}$ junction depth $d$.

\footnotetext{
*Corresponding author. Tel.: + 375296277495 ; fax: + 375172095445 .

E-mail address: fedotov@bsu.by (A. Fedotov).
} 


\section{Solar cell parameters determination at fixed illumination intensity}

There are a number of methods to determine SC parameters based on the analysis of several $I-V$ characteristics of SC measured at different illumination intensities [1-3]. All these methods are developed under an assumption that SC parameters obtained from such measurements $\left(R_{\mathrm{S}}, R_{\mathrm{P}}, A, I_{0}\right)$ do not depend on the illumination intensity. However, such an assumption is not always correct [4]. Therefore the determination of SC parameters from $I-V$ characteristics at a fixed level of excitation is more preferable.

As is known, $I-V$ characteristic with regard to $\mathrm{SC}$ series and parallel resistance can be written as $[4,5]$ :

$$
I=I_{\mathrm{ph}}-I_{0}\left(\exp \left(\frac{e\left(U+I R_{\mathrm{S}}\right)}{A k T}\right)-1\right)-\frac{I R_{\mathrm{S}}+U}{R_{\mathrm{P}}},
$$

where $e$ is the electron charge, $k$ the Boltzman constant, $T$ the temperature, $I$ and $U$ are the load current and voltage, respectively.

It can be assumed that photocurrent $I_{\mathrm{ph}}$ and short-circuit current $I_{\mathrm{SC}}$ are equal to each other, i.e. $I_{\mathrm{SC}} \approx I_{\mathrm{ph}}$. Indeed, the numerical calculation shows that inaccuracy of such an assumption for the typical SC is about $4 \%$ for the illumination conditions of AM1 and $6.5 \%$ at an illumination 10 times greater than AM1 (see Fig. 1).

Eq. (1) can be written for the points $\left(U=0, I=I_{\mathrm{SC}}\right)$ and $\left(U=U_{\mathrm{OC}}, I=0\right)$. Let Eq. (1) be differentiated with respect to voltage for these points. Moreover, let the parameter $A$ be assigned a particular value.

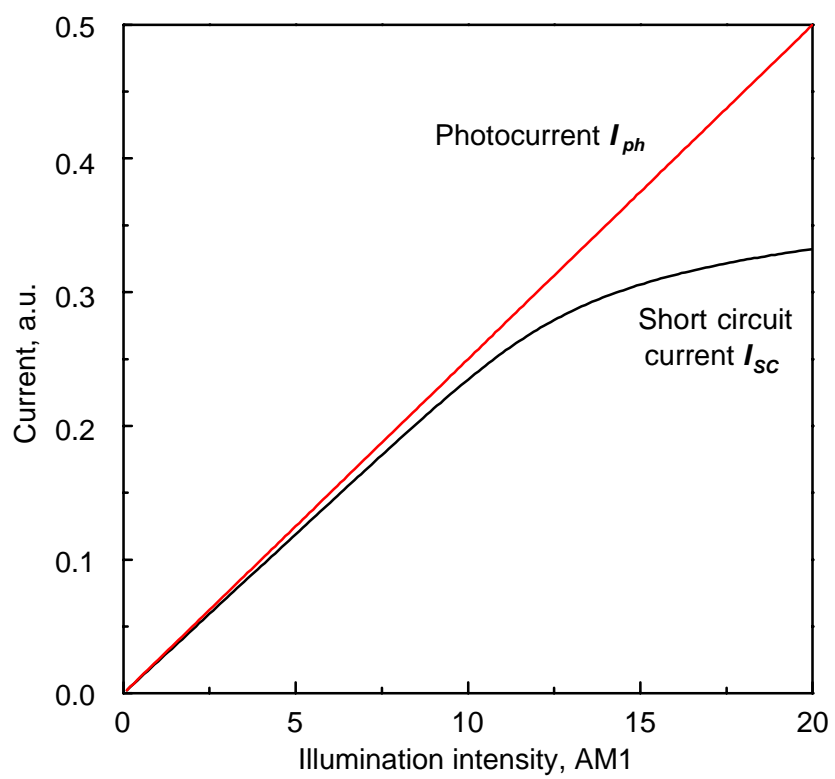

Fig. 1. Dependencies of photocurrent and short-circuit current of SC on the illumination intensity. 
As a result, we obtain four equations:

$$
\begin{aligned}
& 0=-I_{0}\left(\exp \left(\frac{e\left(I_{\mathrm{SC}} R_{\mathrm{S}}\right)}{A k T}\right)-1\right)-\frac{I_{\mathrm{SC}} R_{\mathrm{S}}}{R_{\mathrm{P}}}, \\
& 0=I_{\mathrm{SC}}-I_{0}\left(\exp \left(\frac{e\left(U_{\mathrm{OC}}\right)}{A k T}\right)-1\right)-\frac{U_{\mathrm{OC}}}{R_{\mathrm{P}}}, \\
& \left.\left(I_{U}^{\prime}\right)^{-1}\right|_{U=0}=a=\frac{A k T / e}{-I_{0}+\frac{I_{\mathrm{SC}} R_{\mathrm{S}}}{R_{\mathrm{P}}}-\frac{A k T / \mathrm{e}}{R_{\mathrm{P}}}}-R_{\mathrm{S}}, \\
& \left.\left(I_{U}^{\prime}\right)^{-1}\right|_{I=0}=b=\frac{A k T / e}{-I_{0}-I_{\mathrm{SC}}+\frac{U_{\mathrm{OC}}}{R_{\mathrm{P}}}-\frac{A k T / e}{R_{\mathrm{P}}}}-R_{\mathrm{S}} .
\end{aligned}
$$

Solving this system of equations, we can deduce the following expressions for determination of SC parameters:

$$
\begin{aligned}
& R_{\mathrm{S}}=-\frac{1}{2}\left[\left((a-b)^{2}+\frac{2 q}{I_{\mathrm{SC}}}(a-b)+\left(\frac{U_{\mathrm{OC}}}{I_{\mathrm{SC}}}\right)^{2}\right)^{1 / 2}+(a+b)+\frac{U_{\mathrm{OC}}}{I_{\mathrm{SC}}}\right], \\
& R_{\mathrm{P}}=\frac{U_{\mathrm{OC}}}{\frac{A k T / e}{b+R_{\mathrm{S}}}-\frac{A k T / e}{a+R_{\mathrm{S}}}+I_{\mathrm{SC}}}, \\
& I_{0}=\frac{I_{\mathrm{SC}}}{\gamma}-\frac{U_{\mathrm{OC}}}{\gamma R_{\mathrm{P}}},
\end{aligned}
$$

where

$$
\begin{aligned}
& q=U_{\mathrm{OC}} \frac{\gamma+2}{\gamma}-2 A k T / e, \quad \gamma=\exp \frac{e U_{\mathrm{OC}}}{A k T}-1, \\
& a=\left.\left(I_{U}^{\prime}\right)^{-1}\right|_{U=0}, \quad b=\left.\left(I_{U}^{\prime}\right)^{-1}\right|_{I=0} .
\end{aligned}
$$

In so doing, it is presumed that

$$
1+\frac{R_{\mathrm{S}}}{R_{\mathrm{P}}} \approx 1
$$

The last assumption is quite applicable to a high accuracy for commercial SCs. The values of $U_{\mathrm{OC}}, I_{\mathrm{SC}}$ and $T$ can be determined experimentally. The correctness of the assumed value for parameter $A$ can be proved by its comparison with the one obtained from the next equation:

$$
A=\frac{e}{k T} \frac{\left(U_{\mathrm{M}}-I_{\mathrm{M}} R_{\mathrm{S}}\right)\left(I_{\mathrm{SC}}+I_{0}-I_{\mathrm{M}}-\frac{U_{\mathrm{M}}}{R_{\mathrm{P}}}\right)}{I_{\mathrm{M}}-\frac{U_{\mathrm{M}}}{R_{\mathrm{P}}}},
$$

where $I_{\mathrm{M}}, U_{\mathrm{M}}$ are the current and voltage associated with a maximal power under load. 
Note that there is a possibility of arising of experimental difficulties during investigation of $I-V$ characteristics of high-effective SCs concerning to determination of value of parameter $a=\left.\left(I_{U}^{\prime}\right)^{-1}\right|_{U=0}$ (see (5)). As is known, $I-V$ characteristic curve of high-effective SC is close to straight line parallel to the voltage axis in vicinity of $I_{\mathrm{SC}}$ point. Therefore, frequently it is impossible to determine the slope angle of $I-V$ curve to voltage axis close to $I_{\mathrm{SC}}$ point, i.e.

$$
a \rightarrow-\infty \text {. }
$$

This circumstance creates some impediments in practical using of expression (2) and, consequently, (3) and (4). Let us simplify expression (2) according to condition (6):

$$
\begin{aligned}
R_{\mathrm{S}} & =-\frac{1}{2}\left[\left((a-b)^{2}+\frac{2 q}{I_{\mathrm{SC}}}(a-b)+\left(\frac{U_{\mathrm{OC}}}{I_{\mathrm{SC}}}\right)^{2}\right)^{1 / 2}+(a+b)+\frac{U_{\mathrm{OC}}}{I_{\mathrm{SC}}}\right] \\
& =-\frac{1}{2}\left[|a-b|\left(1+\frac{2 q}{I_{\mathrm{SC}}(a-b)}+\left(\frac{U_{\mathrm{OC}}}{I_{\mathrm{SC}}}\right)^{2} \frac{1}{(a-b)^{2}}\right)^{1 / 2}+(a+b)+\frac{U_{\mathrm{OC}}}{I_{\mathrm{SC}}}\right] \\
& \approx\left|\frac{1}{|a-b|} \ll 1\right| \approx-\frac{1}{2}\left[|a-b|\left(1+\frac{2 q}{I_{\mathrm{SC}}(a-b)}\right)^{1 / 2}+(a+b)+\frac{U_{\mathrm{OC}}}{I_{\mathrm{SC}}}\right] \\
& \approx \mid \begin{array}{l}
(1+x)^{1 / 2} \quad \approx 1+\frac{1}{2} x, \quad x<1 \mid \\
\left.|a|=-a, \quad|b|=-b \quad|\quad| \frac{U_{\mathrm{OC}}}{I_{\mathrm{SC}}}\right]=-\frac{1}{2}\left[-2|b|-\frac{q}{I_{\mathrm{SC}}}+\frac{U_{\mathrm{OC}}}{I_{\mathrm{SC}}}\right] .
\end{array}
\end{aligned}
$$

According to (5), expression for parameter $q$ determination can be rewritten as

$$
q=U_{\mathrm{OC}} \frac{\exp \frac{e U_{\mathrm{OC}}}{A k T}+1}{\exp \frac{e U_{\mathrm{OC}}}{A k T}-1}-2 A k T / e .
$$

For the illumination conditions close to AM1 value $U_{\mathrm{OC}}$ exceeds parameter $\frac{A k T}{e}$ in several times, and so expression (7) can be easily transformed to

$$
q=U_{\mathrm{OC}}-2 A k T / e .
$$

So, under the condition $a \rightarrow-\infty$ the expressions (2-4) look in the following way:

$$
R_{\mathrm{S}}=|b|-\frac{A k T / e}{I_{\mathrm{SC}}}, \quad R_{\mathrm{P}} \rightarrow \infty, \quad I_{0}=\frac{I_{\mathrm{SC}}}{\gamma} .
$$

\section{Determination of the $p-n$ junction depth}

The $\mathrm{p}-\mathrm{n}$ junction depth may be obtained by different methods: low-angle and spherical bevelling [6]; calculation of depth of linearly graded $\mathrm{p}-\mathrm{n}$ junction from the 
known dopant distribution [7]; determination of the $\mathrm{p}-\mathrm{n}$ junction depth from the maximum spectral photoresponse [8]; determination of the recombination parameters from spectral dependencies of SC short-circuit currents under front- and back-side illumination [9]; calculation of the $\mathrm{p}-\mathrm{n}$ junction depth based on its spectral characteristics using variation of the surface recombination velocity [10].

Unfortunately, no methods satisfy the following requirements completely:

- taking into account the construction peculiarities of commercial SCs (small depth of $\mathrm{p}-\mathrm{n}$ junction, the presence of continuous back-side metallization, high doping of the surface layer, etc.);

- nondestructive character.

Below we propose a nondestructive method for the $\mathrm{p}-\mathrm{n}$ junction depth determination which is based on analysis of the spectral dependencies for SC photocurrent at different applied bias voltages and takes into account the abovementioned peculiarities of commercial SCs.

Fig. 2 shows the sketch of different SC regions. Here $W$ is the space charge region (SCR) width, $L_{\mathrm{n}}$ the diffusion length of electrons in p-type region, $L_{\mathrm{p}}$ the diffusion length of holes in n-type region, $x_{j}$ the boundary of SCR in n-type region, $d$ the $\mathrm{p}-\mathrm{n}$ junction depth, $H$ the thickness of quasi-neutral part of p-base and $H^{\prime}$ the total SC thickness.

As is known, the density of photocurrent arising due to the generation of carriers in $\mathrm{SC}$ consists of three components

$$
J=J_{\mathrm{p}}+J_{\mathrm{n}}+J_{\mathrm{SCR}},
$$

where $J_{\mathrm{p}}$ is the current density of holes that are generated in region 1 and arrive at $\mathrm{SCR} ; J_{\mathrm{n}}$ the current density of electrons generated in region 3 and arriving to SCR; $J_{\mathrm{SCR}}$ the current density of carriers generated in SCR (region 2).

The last component of current can be calculated according to

$$
J_{\mathrm{SCR}}=e F(1-R) \exp \left(-\alpha x_{j}\right)[1-\exp (-\alpha W)],
$$

where $F=F(\lambda)$ is the spectral density of photon flux, $R=R(\lambda)$ the reflection coefficient, $\alpha=\alpha(\lambda)$ the absorbtion coefficient and $\lambda$ the wavelength.

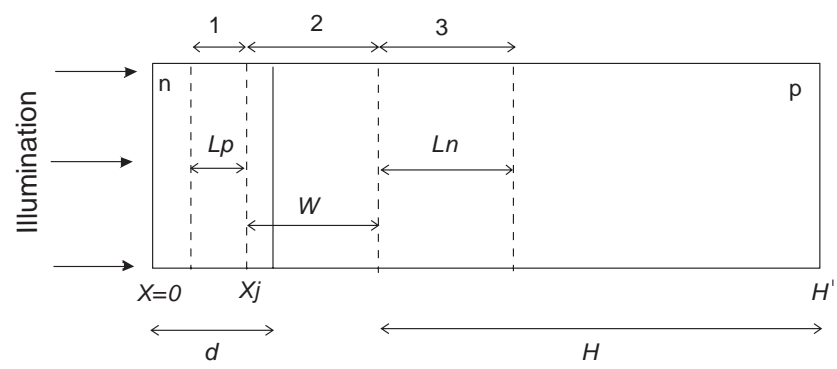

Fig. 2. Sketch of critical SC regions. 
The width of SCR can be expressed as

$$
W=z\left(U_{0}+U\right)^{1 / 2},
$$

where $U_{0}$ is the built-in potential of $\mathrm{p}-\mathrm{n}$ junction and $U$ the applied voltage.

Let us assume that $z$ is a constant that is independent of the SCR width. In reality, this is true for the depletion region with constant dopant concentration, i.e. Eq. (8) is applicable to the abrupt $\mathrm{p}-\mathrm{n}$ junction only. As is known, for the abrupt $\mathrm{p}-\mathrm{n}$ junction with highly doped n-region one can write

$$
x_{\mathrm{p}} \gg x_{\mathrm{n}},
$$

where $x_{\mathrm{p}}$ and $x_{\mathrm{n}}$ are the widths of SCR in $\mathrm{p}$ - and n-regions, respectively.

We analyze the dopant distribution in more realistic linearly graded $\mathrm{p}-\mathrm{n}$ junction of SC. As seen from Fig. 3, practically the distribution of a noncompensated dopant in $\mathrm{p}$-region of the linearly graded $\mathrm{p}-\mathrm{n}$ junction corresponds to that in case of the abrupt p-n junction. Therefore, Eq. (8) can be used with high precision for description of the linearly graded $\mathrm{p}-\mathrm{n}$ junctions in SCs.

Hence, by virtue of $x_{\mathrm{p}} \gg x_{\mathrm{n}}$ we have

$$
\left(x_{j}\right)_{W}^{\prime}=0 \text {, }
$$

that in its turn gives

$$
\left(J_{\mathrm{p}}\right)_{W}^{\prime}=0 .
$$

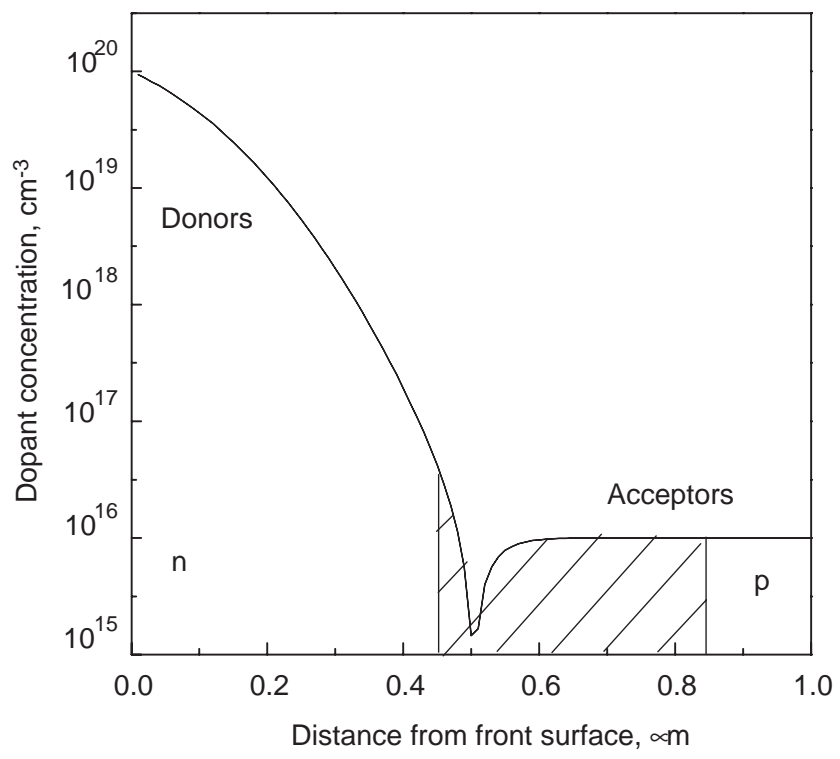

Fig. 3. Calculated distribution of dopants in typical commercial SC. 
Now we obtain the expression for $J_{\mathrm{n}}$. At low excitation level the $1 \mathrm{D}$ steady-state continuity equation for electrons in p-type region can be written as follows:

$$
g_{\mathrm{n}}-\frac{D_{\mathrm{n}}}{L_{\mathrm{n}}^{2}}\left(n_{\mathrm{p}}-n_{\mathrm{p} 0}\right)+\frac{1 \mathrm{~d} J_{\mathrm{n}}}{e}=0,
$$

where $n_{\mathrm{p}}$ is the total electron concentration, $n_{\mathrm{p} 0}$ the equilibrium electron concentration, $D_{\mathrm{n}}$ the diffusion coefficient of electrons and $g_{\mathrm{n}}$ the generation rate of electrons.

The current density of electrons is defined by expression

$$
J_{\mathrm{n}}=e \mu_{\mathrm{n}} n_{\mathrm{p}} E+e D_{\mathrm{n}} \frac{\mathrm{d} n_{\mathrm{p}}}{\mathrm{d} x}
$$

where $\mu_{\mathrm{n}}$ is the electron mobility and $E$ the electric field intensity.

We introduce the last expression into the continuity equation and suppose that $E=0$. Indeed, the gradient of a noncompensated dopant in the p-type region is negligibly small (see Fig. 3). As a result, we can write

$$
D_{\mathrm{n}} \frac{\mathrm{d}^{2} n_{\mathrm{p}}}{\mathrm{d} x^{2}}-\frac{D_{\mathrm{n}}}{L_{\mathrm{n}}^{2}}\left(n_{\mathrm{p}}-n_{\mathrm{p} 0}\right)+\alpha F(1-R) \mathrm{e}^{-\alpha x}=0 .
$$

Hence

$$
J_{\mathrm{n}}=e F(1-R) D_{\mathrm{n}}\left(\frac{\mathrm{d} n_{\mathrm{p}}}{\mathrm{d} x}\right)_{x_{j}+W} .
$$

As was shown in Ref. [11], with the use of the boundary conditions

$$
\begin{aligned}
& n_{\mathrm{p}}=\left.n_{\mathrm{p} 0}\right|_{x=x_{j}+W}, \\
& S_{\mathrm{n}}\left(n_{\mathrm{p}}-n_{\mathrm{p} 0}\right)=-\left.D_{\mathrm{n}} \frac{\mathrm{d} n_{\mathrm{p}}}{\mathrm{d} x}\right|_{x=H^{\prime}},
\end{aligned}
$$

( $S_{\mathrm{n}}$ is the velocity of surface recombination of electrons on the back-side surface of $\mathrm{SC}$ ), one can obtain the next expression for the electron current density:

$$
\begin{aligned}
J_{\mathrm{n}}= & e \frac{F(1-R) \alpha L_{\mathrm{n}}}{\alpha^{2} L_{\mathrm{n}}^{2}-1} \exp \left(-\alpha\left(x_{j}+W\right)\right) \\
& \times\left[\alpha L_{\mathrm{n}}-\frac{\left(\frac{S_{\mathrm{n}}}{D_{\mathrm{n}}} L_{\mathrm{n}}\right)\left(\operatorname{ch} \frac{H^{\prime}}{L_{\mathrm{n}}}-\exp \left(-\alpha H^{\prime}\right)\right)+\operatorname{sh} \frac{H^{\prime}}{L_{\mathrm{n}}}+\alpha L_{\mathrm{n}} \exp \left(-\alpha H^{\prime}\right)}{\left(\frac{S_{\mathrm{n}}}{D_{\mathrm{n}}} L_{\mathrm{n}}\right) \operatorname{sh} \frac{H^{\prime}}{L_{\mathrm{n}}}+\operatorname{ch} \frac{H^{\prime}}{L_{\mathrm{n}}}}\right] .
\end{aligned}
$$

Let us impose the following restriction:

$$
\begin{aligned}
& \alpha H^{\prime} \gg 1 \Rightarrow \exp \left(-\alpha H^{\prime}\right) \approx 0, \\
& \exp \left(\frac{H^{\prime}}{L_{\mathrm{n}}}\right) \gg \exp \left(-\frac{H^{\prime}}{L_{\mathrm{n}}}\right) .
\end{aligned}
$$


The requirements of Eqs. (10) and (11) imply that the proposed method is applicable for "thick" cells, i.e. for the cells where we can neglect a part of the carriers which arrive at the back-side contact of the illumination corresponding to the fundamental absorption edge.

Taking into account Eqs. (10) and (11), we obtain

$$
\begin{aligned}
J_{\mathrm{n}} & =e \frac{F(1-R) \alpha L_{\mathrm{n}}}{\alpha^{2} L_{\mathrm{n}}^{2}-1} \exp \left(-\alpha\left(x_{j}+W\right)\right)\left[\alpha L_{\mathrm{n}}-\frac{\left(\frac{S_{\mathrm{n}}}{D_{\mathrm{n}}} L_{\mathrm{n}}\right) \exp \left(\frac{H^{\prime}}{L_{\mathrm{n}}}\right)+\exp \left(\frac{H^{\prime}}{L_{\mathrm{n}}}\right)}{\left.\left(\frac{S_{\mathrm{n}}}{D_{\mathrm{n}}} L_{\mathrm{n}}\right) \exp \left(\frac{H^{\prime}}{L_{\mathrm{n}}}\right)+\exp \left(\frac{H^{\prime}}{L_{\mathrm{n}}}\right)\right]}\right. \\
& =e \frac{F(1-R) \alpha L_{\mathrm{n}}\left(\alpha L_{\mathrm{n}}-1\right)}{\alpha^{2} L_{\mathrm{n}}^{2}-1} \exp \left(-\alpha\left(x_{j}+W\right)\right) \\
& =e \frac{F(1-R) \alpha L_{\mathrm{n}}}{\alpha L_{\mathrm{n}}+1} \exp \left(-\alpha\left(x_{j}+W\right)\right) .
\end{aligned}
$$

Thus, current density can be written as follows

$$
\begin{aligned}
J= & J_{\mathrm{p}}+e F(1-R) \exp \left(-\alpha x_{j}\right)(1-\exp (-\alpha W)) \\
& +e \frac{F(1-R) \alpha L_{\mathrm{n}}}{\alpha L_{\mathrm{n}}+1} \exp \left(-\alpha\left(x_{j}+W\right)\right) .
\end{aligned}
$$

Measuring experimentally the current values at different applied bias voltages, we can obtain the relation

$$
\begin{aligned}
J 1-J 2= & e F(1-R) \exp \left(-\alpha x_{j}\right)[\exp (-\alpha W 2)-\exp (-\alpha W 1)] \\
& -e \frac{F(1-R) \alpha L_{\mathrm{n}}}{\alpha L_{\mathrm{n}}+1} \exp \left(-\alpha x_{j}\right)[\exp (-\alpha W 2)-\exp (-\alpha W 1)],
\end{aligned}
$$

and determine the parameter $x_{j}$ from the equation as follows:

$$
x_{j}=\frac{1}{\alpha} \ln \left(\frac{e F(1-R)}{1+\alpha L_{\mathrm{n}}} \frac{\exp (-\alpha W 2)-\exp (-\alpha W 1)}{J 1-J 2}\right) .
$$

Here $J 1$ and $J 2$ are current densities for two different values of the applied bias voltage, $W 1$ and $W 2$ the corresponding values of the SCR width.

The parameter $z$ can be found from the following equation:

$$
\frac{J 2-J 3}{J 1-J 2}-\frac{\exp \left(-\alpha z\left(U_{0}+U_{2}\right)^{1 / 2}\right)-\exp \left(-\alpha z\left(U_{0}+U_{3}\right)^{1 / 2}\right)}{\exp \left(-\alpha z\left(U_{0}+U_{1}\right)^{1 / 2}\right)-\exp \left(-\alpha z\left(U_{0}+U_{2}\right)^{1 / 2}\right)}=0,
$$

where $J 1, J 2$ and $J 3$ are the current densities corresponding to the applied bias voltages $U_{1}, U_{2}$ and $U_{3}$, respectively.

Let us assume that $d=x_{j}$, then we obtain the next expression for the $\mathrm{p}-\mathrm{n}$ junction depth:

$$
d=\frac{1}{\alpha} \ln \frac{e F(1-R)\left(\exp \left(-\alpha z\left(U_{0}+U_{2}\right)^{1 / 2}\right)-\exp \left(-\alpha z\left(U_{0}+U_{1}\right)^{1 / 2}\right)\right)}{\left(\alpha L_{\mathrm{n}}+1\right)\left(J_{1}-J_{2}\right)} .
$$

It is seen from Eq. (12) that the determination of the $\mathrm{p}-\mathrm{n}$ junction depth requires knowledge of the value of the diffusion length of electrons. To determine $L_{\mathrm{n}}$ one can use the procedure described in Ref. [12]. 


\section{Conclusion}

In this work, methods for the determination of different solar cell parameters are developed. The calculation of series and parallel resistance, diode coefficient and reverse current density of $\mathrm{SC}$ is based on the analysis of its current-voltage characteristic at fixed illumination intensity. As is shown, the $\mathrm{p}-\mathrm{n}$ junction depth can be determined from the measurements of spectral dependencies for the diode photocurrent at different values of the applied bias voltage.

\section{References}

[1] N. Bordin, A. Saitzeva, V. Strel'zova, Sov. Heliotech. 1 (1977) 17-23.

[2] A.P. Gorban, Sov. Optoelectron. Semiconduct. Tech. 9 (1991) 85-90.

[3] R.A. Sinton, A. Cuevas, Proceedings of the 16th Euro PV Solar Energy Conference, Glasgow, England, 1-5 May, 2000.

[4] A.L. Fahrenbrugh, R.H. Bube, Fundamentals of Solar Cells: Photovoltaic Solar Energy Conversion, Academic Press, New York, 1983.

[5] M.A. Green, Silicon Solar Cells: Advanced Principles and Practice, University of South Wales, Sydney, 1995.

[6] V.V. Batavin, Yu.A. Konzevoi, Yu.V. Fedorovitch, Measurement of Parameters of Semiconductor Materials and Structures, Radio I Svyaz', Moscow, 1985.

[7] N.A. Poklonski, N.I. Gorbatchuk, S.B. Lastovski, Low Dimensional Systems, Belarusian State University, Minsk, Belarus, 2000, pp. 74-80.

[8] M.B. Kagan, T.L. Lyubashevskaya, Sov. Heliotech. 4 (1968) 11-15.

[9] Yu.A. Konzevoi, Zavodskaya Lab. 66 (2000) 32-33 (in Russian).

[10] V.K. Subashiev, G.B. Dubrovski, V.A. Petrusevitch, Sov. Phys.-Solid State 2 (1960) 1978-1980.

[11] S.M. Sze, Physics of Semiconductor Devices, Wiley-Interscience Publication, New York, 1981.

[12] D.L. Lile, N.M. Davis, Solid State Electron. 18 (1975) 699-704. 